\title{
Immunotherapy response evaluation with magnetic resonance elastography (MRE) in advanced HCC
}

\author{
Aliya Qayyum", Ken-Pin Hwang ${ }^{2}$, Jason Stafford², Anuj Verma ${ }^{3}$, Dipen M. Maru ${ }^{4}$ Subramanya Sandesh ${ }^{3}$, Jia Sun ${ }^{5}$, \\ Roberto Carmagnani Pestana ${ }^{6}$, Rony Avritscher ${ }^{7}$, Manal M. Hassan ${ }^{8}$, Hesham Amin $^{9}$, Asif Rashid ${ }^{4}$, \\ Ignacio I. Wistuba ${ }^{3}$, Richard L. Ehman ${ }^{10}$, Jingfei $\mathrm{Ma}^{2}$ and Ahmed O. Kaseb ${ }^{6^{*}}$
}

\begin{abstract}
Background: Currently, there are no imaging predictors of immunotherapy outcome in hepatocellular carcinoma (HCC). The study aim was to determine if stiffness changes measured by magnetic resonance elastography (MRE) can be a predictor of immunotherapy response in patients with advanced HCC.

Materials and methods: This was a prospective study of 15 patients with biopsy proven-advanced HCC treated with Pembrolizumab. All patients had liver MRE and liver biopsy at baseline and at 6 weeks of therapy. Change in HCC stiffness on MRE was compared with overall survival (OS), time to disease progression (TTP), and number of intratumoral CD3+ T lymphocytes. Analysis was performed using descriptive statistics and Spearman correlation (R); $p$-value $<0.05$ was considered statistically significant.

Results: Nine patients were evaluable. Median age was 71 years (range, 54-78). Etiology of liver disease was HCV $(n=4), \mathrm{HBV}(n=1)$ and NASH $(n=4)$. Median OS and TTP were 44 weeks and 13 weeks, respectively. Average baseline HCC stiffness and change in HCC stiffness were $5.0 \mathrm{kPa}$ and $0.12 \mathrm{kPa}$, respectively. In contrast, average nontumor liver stiffness was $3.2 \mathrm{kPa}$, and did not significantly change at 6 weeks $(p=0.42)$. Average size of measured tumor and change in size were $4 \mathrm{~cm}$ and $-0.32 \mathrm{~cm}$, respectively. Change in HCC stiffness at 6 weeks correlated significantly with OS $(R=0.81)$, and TTP $(R=0.88, p<0.01)$. Abundance of intratumoral T lymphocytes on tumor biopsy correlated significantly with HCC stiffness $(R=0.79, p=0.007)$.
\end{abstract}

Conclusion: Our pilot MRE data suggests early change in tumor stiffness may be an indicator of immunotherapy response in patients with advanced HCC.

Keywords: Hepatocellular carcinoma, Magnetic resonance Elastopgraphy, Immunotherapy

\section{Introduction}

HCC is considered the fifth most common malignancy worldwide, with the third-highest mortality [1]. An estimated that $80 \%$ of patients present with advanced stage tumor not amenable to curative therapy $[1,2]$. Oral tyrosine kinase inhibitor (sorafenib) has been the frontline standard of care since 2007 for treatment of advanced HCC with preserved liver

\footnotetext{
* Correspondence: aqayyum@mdanderson.org; akaseb@mdanderson.org 'Department of Abdominal Imaging, UT MD Anderson Cancer Center, 1400 Pressler Street, Houston, Texas, USA

${ }^{6}$ Department of GI Medical Oncology, UT MD Anderson Cancer Center, Houston, USA

Full list of author information is available at the end of the article
}

function [3]. Newer systemic treatments with immunotherapy agents are being investigated, such as Nivolumab and Pembrolizumab (anti-PD-1 mAb) which enhance immune function and cytotoxic $\mathrm{T}$ lymphocyte (CTL)-mediated immune response against cancer cells [4-6].

Imaging assessment of HCC response to targeted therapies is challenging since reduction in size may not occur. Tumor stability is used as a marker of response without necessarily conferring improved outcomes [7-9]. MR Elastography (MRE) is a relatively novel technique, and has been shown to be superior to ultrasound-based transient elastography for 
assessment of liver fibrosis [10]. MRE may be used to distinguish malignant from benign liver tumors [11], which is thought be due to the abnormal cellular microenvironment of neoplastic conditions, including denser extracellular matrix, increase cellularity, vascularity, and interstitial pressure, causing increased stiffness.

Immunotherapy response decreases viable tumor cells, but increases immune content, and causes stromal and fibrosis flux due to effects on immune cell function. We hypothesize that such changes in tumor cellularity and stroma in patients treated with anti-PD-1 immunotherapy would effect MRE tumor stiffness. The purpose of our study was to determine if stiffness changes measured by magnetic resonance elastography (MRE) can be a predictor of immunotherapy response in patients with advanced HCC.

\section{Materials and methods}

This was a prospective, Institutional Review Board approved study. A total of 15 patients were accrued through our Liver Center, with biopsy proven advanced HCC (not amenable to curative therapy), Child-Pugh Score A, who were treated with anti-PD1, Pembrolizumab monotherapy. Clinical responses were determined by blinded independent review using RECIST 1.1 and mRECIST 1.1. All patients provided written informed consent. Eligible patients were over 18 years of age with radiographic disease progression on sorafenib or intolerance to sorafenib treatment, and ECOG 0 or 1 . All patients underwent liver MRI with MR Elastography (MRE) and liver biopsy at baseline and at 6 weeks of therapy. Date of documented disease progression on patient follow-up and date of death were obtained from the patients' electronic medical records.

MR Elastography (MRE) was performed on a $3 \mathrm{~T}$ whole body MRI scanner (Discovery $750 \mathrm{HD}$; GE Healthcare, Waukesha, WI) with a 32-channel phased-array torso coil. Acoustic waves at $60 \mathrm{~Hz}$ were generated by an active driver and transmitted to the liver through an external passive driver placed over the subject's right upper quadrant (overlying the liver). Data was acquired using a 2Dechoplanar imaging (EPI) based MRE sequence with the following imaging parameters: TR/TE $=600 \mathrm{~ms} /$ Minimum Full; slice thickness $/$ gap $=7 / 2.5 \mathrm{~mm}$; FOV $38-42 \mathrm{~cm}$; acquisition matrix $=64 \times 64 ; \mathrm{NEX}=2 ; 6$ axial slices through widest cross-section of the liver including at least one slice through the tumor; parallel imaging factor $=2$, and acquisition time $=16-19 \mathrm{~s}$ (one breath hold). Automated in-line post-processing was used to generate quantitative maps or "elastograms" of liver stiffness in units of kilopascals $(\mathrm{kPa})$ [12-16], and a mask overlay to exclude pixels with low inversion processing confidence [12].

\section{Image analysis}

Liver and tumor stiffness $(\mathrm{kPa})$ was measured by an independent reader blinded to pathologic and clinial data. MRE derived average non-tumorous liver stiffness $(\mathrm{kPa})$ was measured by placing regions-ofinterest (ROIs) on the MRE elastograms (stiffness maps) to include as much of the non-tumorous liver as possible away from the HCC. Measurements were obtained at 3 axial levels through widest portions of liver while excluding any visible major vessels [12-16]. Average HCC stiffness was measured by drawing ROIs on the elastograms covering as much of the HCC as possible. HCC size and extent of tumor enhancement were also recorded. HCC enhancement was categorized on arterial phase images using a 4-point scale (0-3), with no enhancement as $0,<25 \%$ as $1,25-50 \%$ as 2 and $>50 \%$ as 3 .

\section{Pathology analysis}

All subjects underwent image guided liver biopsy at baseline and at 6 weeks of pembrolizumab treatment. Histopathology analysis was performed by a pathologist in five intratumoral areas using $660 \mu \mathrm{m} \times 500 \mu \mathrm{m}$ $\left(0.33 \mathrm{~mm}^{2}\right)$ region of interest (ROI) at $\times 20$ magnification to cover a total intratumoral area of $1.65 \mathrm{~mm}^{2}$. A pancytokeratin (AE1/AE3) marker was used and the intratumoral area compartmentalized in epithelial (tumor) and stroma compartment. Total intratumoral lymphocytes $\left(\mathrm{CD}^{+}\right)$was expressed as an average of cell densities from the areas analyzed $\left(\mathrm{n} / \mathrm{mm}^{2}\right)$. HCC tumor grade and number of T lymphocytes $\left(\mathrm{CD}^{+} ; \mathrm{n} /\right.$ $\mathrm{mm} 2$ ) were obtained from the database of the parent MDACC-sponsored clinical protocol supported by Merck \& Co.

\section{Statistical analysis}

Summary of demographic and clinical characteristics were provided in percentages, means, medians, standard deviations (SD), and range. Spearman rank correlation was estimated between imaging measurements at the 2

Table 1 Correlation of imaging and pathology with with overall survival; ( $\mathrm{R}$, Spearman correlation coefficient; kPa, kilopascals)

\begin{tabular}{|c|c|c|c|c|}
\hline Variable & Average & Range & $\begin{array}{l}\text { Correlation with } \\
\text { Overall Survival (R) }\end{array}$ & $P$-value \\
\hline $\begin{array}{l}\text { Baseline HCC } \\
\text { size }(\mathrm{cm})\end{array}$ & 4.0 & $1.5-8.5$ & 0.32 & 0.4 \\
\hline $\begin{array}{l}\text { Change in } \mathrm{HCC} \\
\text { size }(\mathrm{cm})\end{array}$ & -0.32 & {$[-2.2]-0.4$} & 0.21 & 0.58 \\
\hline $\begin{array}{l}\text { Baseline HCC } \\
\text { stiffness (kPa) }\end{array}$ & 5.0 & $2.4-9.1$ & -0.66 & 0.055 \\
\hline $\begin{array}{l}\text { Change in } \mathrm{HCC} \\
\text { stiffness }(\mathrm{kPa})\end{array}$ & 0.12 & {$[-2.1]-2.8$} & 0.81 & 0.008 \\
\hline $\begin{array}{l}\text { Non-tumor liver } \\
\text { stiffness (kPa) }\end{array}$ & 3.2 & $2.1-4.3$ & -0.65 & 0.056 \\
\hline
\end{tabular}

Bold face type of numbers denotes findings are statistically significant 

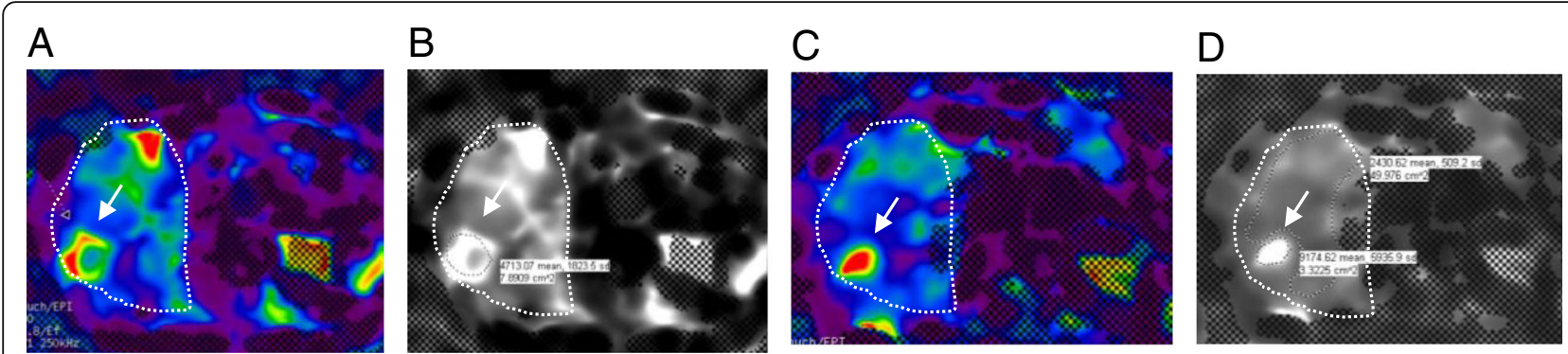

Fig. 1 Elastogram color map. a gray scale $\mathbf{b}$ at baseline showing HCC (arrow). HCC stiffness increased on as indicated by increased red color of the tumor ( $\mathbf{c}$ and $\mathbf{d}$ ). Liver is demarcted by hashed lines (----)

time-points, and between imaging measurements and lymphocytes $(\mathrm{n} / \mathrm{mm} 2)$ on pathology. Overall survival (OS) or time-to-progression (TTP) was correlated with baseline and change in both HCC size and stiffness, and baseline non-tumorous liver stiffness. Comparison of tumor grade with HCC stiffness, and of changes in HCC stiffness between groups with survival of either more than or less than 52 weeks was performed using Wilcoxon rank-sum test.

HCC stiffness was correlated with $\mathrm{HCC}$ size at baseline and tumor $\mathrm{T}$ lymphocytes. A scatter plot with a linear regression line was used to show the relationship between HCC stiffness difference and overall survival time (or time to progression). All tests were two-sided and $p$-values of 0.05 or less were considered statistically significant. Statistical analyses were carried out using SAS version 9.3 and JMP version 14.0 (SAS Institute, Cary, NC).

\section{Results}

Of the total of 15 patients, 4 withdrew ( 2 died before the followup MRE scan could be performed, 1 patient decided on hospice care and declined further treatment; 1 patient was intolerant to treatment), 1 patient did not undergo MRE scan, and 1 patient had MRE exam failure. The remaining 9 patients included 6 men. Median age was 71 years (range, 54-78).

HCC was well-differentiated in 2 of 9 patients, moderately differentiated in 6 and poorly differentiated in 1 . Median overall survival and time to progression were 44 weeks (range, 16-70) and 13 weeks (range, 9-48), respectively. Etiology of liver disease was $\operatorname{HCV}(n=4)$, $\operatorname{HBV}(n=1)$ and NASH $(n=4)$.

No correlation was found for stiffness of non-tumor liver and etiology of liver disease.

Average non-tumor liver stiffness was $3.2 \mathrm{kPa}$ (range, 2.1-4.3), and did not significantly change at 6 weeks $(p=0.42)$. Baseline non-tumor liver stiffness did not significantly correlate with overall survival, $(p=0.056)$, Table 1.
Seven of 9 HCC demonstrated $>50 \%$ enhancement at baseline, 1 demonstrated $20-50 \%$ and $1<25 \%$. Decrease in $\mathrm{HCC}$ enhancement category was only seen in 2 of 9 patients at 6 weeks.

Correlation of overall survival with baseline and change in HCC size and stiffness are shown in Table 1. Average HCC size and change in size were $4 \mathrm{~cm}$ (range, 1.5-8.5) and - 0.32 (range, [-2.2] - 0.4), respectively. There was no significant correlation between overall survival and baseline HCC size $(p=0.4)$.

Average baseline HCC stiffness and change in stiffness were $5 \mathrm{kPa}$ (range, 2.4-9.1) and $0.12 \mathrm{kPa}$ (range, $[-2.1]-2.8)$, respectively. There was no significant correlation between overall survival and baseline HCC stiffness $(p=0.055)$, Table 1 . Increase in HCC stiffness on follow-up imaging (Fig. 1a and b) was seen in 5 patients, decrease in 3 patients and no change in 1 patient (Table 2). Increase in HCC stiffness at 6 weeks correlated significantly with overall survival $(R=0.81, p=0.008)$, Fig. 2a, and with survival of more than 52 weeks from start of therapy $(p=0.02)$, Fig. 2b. Increase in HCC stiffness at 6 weeks also correlated significantly with time to progression $(R=0.88, p=0.009)$, Fig. $2 \mathrm{c}$.

HCC stiffness was significantly correlated with baseline HCC size $(R=0.7, p=0.036)$, but not with tumor

Table 2 MRE HCC stiffness (kilopascals, kPa) at baseline and 6 weeks with overall survival (OS) and time-to-progression (TTP)

\begin{tabular}{llllll}
\hline Patient & $\begin{array}{l}\text { Baseline HCC } \\
\text { stiffness }(\mathrm{kPa})\end{array}$ & $\begin{array}{l}\text { 6 week HCC } \\
\text { stiffness }(\mathrm{kPa})\end{array}$ & $\begin{array}{l}\text { Difference in } \\
\text { stiffness }(\mathrm{kPa})\end{array}$ & $\begin{array}{l}\text { OS } \\
\text { (weeks) }\end{array}$ & $\begin{array}{l}\text { TTP } \\
\text { (weeks) }\end{array}$ \\
\hline 1 & 9.1 & 7 & -2.1 & 24 & 9 \\
2 & 2.8 & 3.9 & 1.1 & 70 & 48 \\
3 & 7.0 & 6.2 & -0.8 & 16 & - \\
4 & 3.3 & 3.3 & 0 & 44 & 10 \\
5 & 4.2 & 5.6 & 1.4 & 65 & 40 \\
6 & 2.4 & 5.2 & 2.8 & 52 & - \\
7 & 6.1 & 5.0 & -1.1 & 35 & 13 \\
8 & 6.7 & 7.6 & 0.9 & 52 & 17 \\
9 & 3.8 & 2.7 & -1.1 & 35 & 10 \\
\hline
\end{tabular}




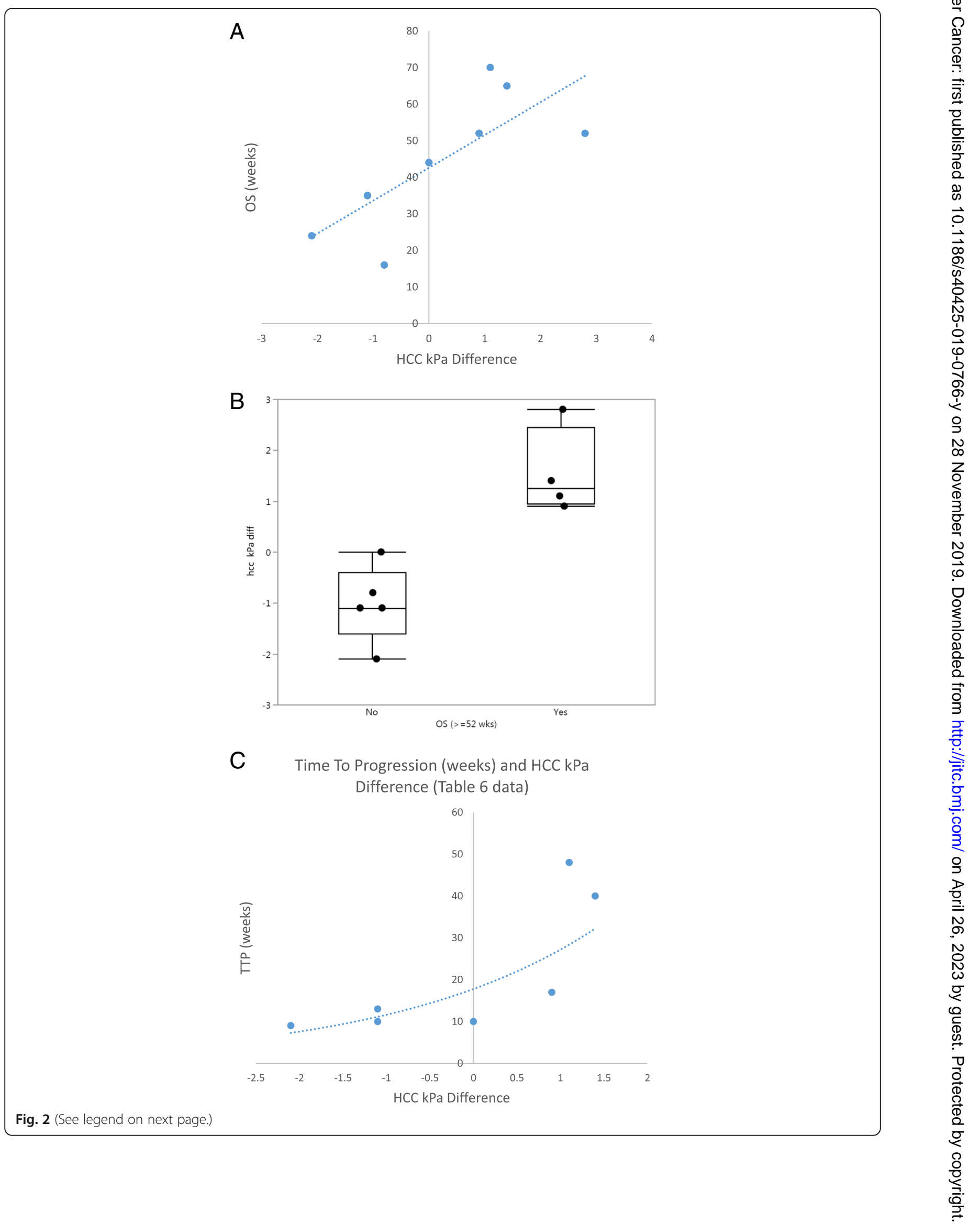


(See figure on previous page.)

Fig. 2 Association between MRE parameters and survival. a HCC stiffness difference (kilopascals, $\mathrm{kPa}$ ) between baseline and 6 week MRE, correlated significantly with overall survival (OS), (Spearman $R=0.88, p<0.05$ ); b A greater increase in HCC stiffness (kilopascals, $\mathrm{kPa}$ ) was significantly associated with a survival of more than 52 weeks from start of therapy, $p=0.02$; $\mathbf{c}$ HCC stiffness difference (kPa) between baseline and 6 week MRE, correlated significantly with time-to-progression (weeks), (Spearman $R=0.88, p=0.009 ; n=7$ )

grade $(p=0.3)$. HCC stiffness was significantly correlated with tumor $\mathrm{T}$ lymphocytes $(R=0.79, p<0.01)$ Fig. 3, however, pre-and post tumor lymphocyte evaluation was not available in all cases.

\section{Discussion}

Imaging predictors of immunotherapy response in $\mathrm{HCC}$ could aid the identification of patients more likely to benefit from the treatment and represent an important unmet need. Our preliminary data suggests that an early increase in $\mathrm{HCC}$ stiffness may be an indicator of early immunotherapy (anti-PD-1) response.

There is limited data on MRE assessment of HCC. Thompson et al. [17], reported a trend toward increased HCC stiffness in well-moderately differentiated compared to poorly differentiated HCC but no correlation with size $(n=21)$. Our findings are concordant with this study since we also did not observe a significant correlation between tumor stiffness and tumor grade. However, this could have been due to there only being 1 patient with poorly differentiated HCC in our study. We observed a correlation between HCC stiffness and HCC size $(p=0.036)$, which is discordant with Thompson et al. [17]. This may have been related to differences in HCC stiffness measurement technique. Thompson et al. [17], only included solid portions of the tumor, but we included as much of the tumor as possible. Furthermore, it is

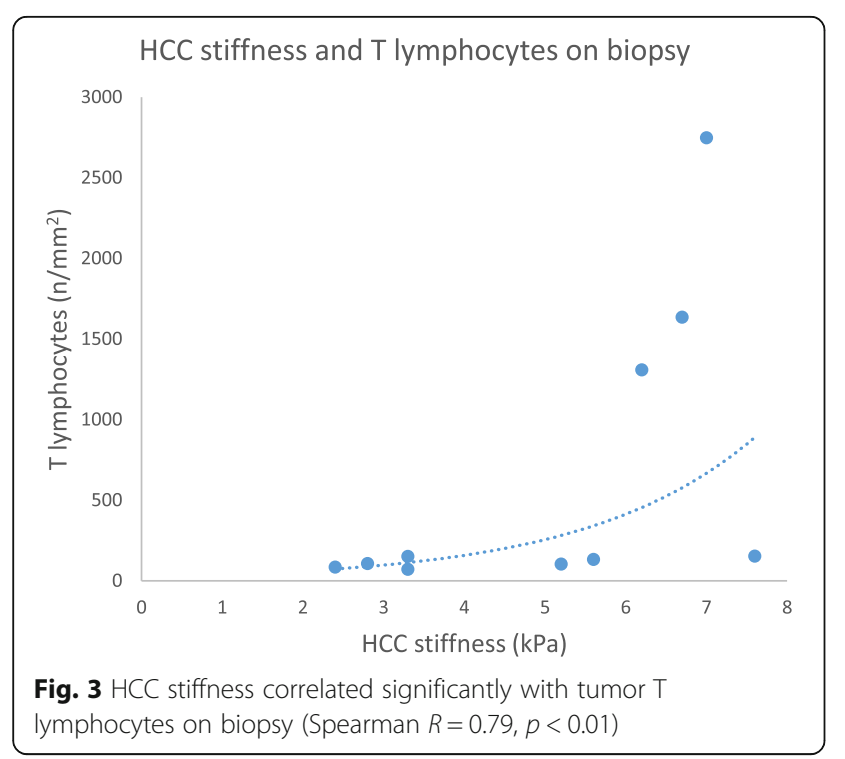

possible that measurements of smaller tumors are assocated with partial volume averaging from the adjacent liver. Larger studies stratifying stiffness measurements with respect to tumor size are needed to understand the relationship between thesee observations.

To our knowledge, there are no prior studies evaluating HCC stiffness changes using MRE in patients treated with immunotherapy. Interestingly, increase in HCC stiffness at 6 weeks was correlated with improved outcome but not change in HCC size or enhancement. Our findings suggest effects related to immune cell infiltration and alteration in tumor stroma (including fibrosis and angiogenesis), can result in early increased tumor stiffness as an independant biomarker of response.

We found a significant correlation between HCC stiffness on MRE and the number of lymphocytes on tumor biopsy. This is supportive of the theory that anti-PD-1 therapy causes increased $\mathrm{T}$ lymphocyte activation in the immune-mediated response to tumor. However, further larger studies are necessary to better understand the underlying mechanisms. If our findings are confirmed, MRE would have an important clinical impact on the response assessment of advanced HCC treatment with checkpoint inhibitors, enabling early identification of treatment response.

Our study has some limitations. First, this is a pilot study that is meant to be hypothesis-generating, and the number of patients is small. Nevertheless, our study showed a strong correlation between early increase in tumor stiffness on MRE and overall survival. Further larger studies are necessary to validate our initial promising observations. Second, HCC was sampled with imageguide core biopsies. Given the heterogeneity of HCC, the biopsy sample may not have been representative of the whole tumor. However, we did observe a correlation between HCC stiffness and tumor T lymphocytes. Third, since patients were being treated with pembrolizumab, they had previously failed treatment or were intolerant to sorafenib. Prior treatment may have affected patient outcomes. However, anti-PD-1 therapy was used as a second-line treatment for all the subjects. Our study focused on change in HCC stiffness on serial MRE, and showed all patients that had an increase in tumor stiffness had better outcomes irrespective of baseline HCC stiffness. Fourth, in our exploratory study HCC stiffness measurements were made by a single independent radiologist placing region-of-interest on the tumor. Future 
larger studies could benefit having more than 1 radiologist measure tumor stiffness to allow assessment of interobserver agreement.

In summary, our preliminary data demonstrated that HCC stiffness increase on MRE in patients treated with immunotherapy significantly correlated with overall survival and time to progression. MRE has the potential to be a useful tool in the assessment of anti-PD-1 therapy in advanced HCC and may be beneficial to the many active immunotherapy trials.

\section{Conclusion}

Our pilot MRE data suggests early change in tumor stiffness may be an indicator of immunotherapy response in patients with advanced HCC.

\section{Abbreviations}

HCC: Hepatocellular carcinoma; MRE: Magnetic Resonance Elastograpy

\section{Acknowledgements}

Not applicable.

\begin{abstract}
Authors' contributions
All authors contributed significantly to the final manuscript. AQ and AOK contributed to the study design, data collection and analysis and writing of the manuscript; K-PH, JS, AV, DMM, SS, RPC, RA, HA, RA, IIW, RLE and JM contributed to the study design, data collection, and writing of the manuscript; $R C P$, contributed to the study design and writing of the manuscript; RA, JS and $\mathrm{MMH}$, contributed to the study design, statistical analysis and writing of the manuscript. All authors read and approved the final manuscript.
\end{abstract}

\section{Funding}

Support for this study was provided by Merck Sharp \& Dohme Corp., a subsidiary of Merck \& Co., Inc., Kenilworth, NJ, USA.

\section{Availability of data and materials}

The datasets used and/or analyzed during the current study are available from the corresponding author on reasonable request.

\section{Ethics approval and consent to participate}

This was a prospective, Institutional Review Board approved study. All patients provided written informed consent.

\section{Consent for publication}

Not applicable.

\section{Competing interests}

The authors declare that they have no competing interests.

\section{Author details}

${ }^{1}$ Department of Abdominal Imaging, UT MD Anderson Cancer Center, 1400 Pressler Street, Houston, Texas, USA. ²Department of Imaging Physics, UT MD Anderson Cancer Center, Houston, Texas, USA. ${ }^{3}$ Department of Translational Molecular Pathology, UT MD Anderson Cancer Center, Houston, Texas, USA. ${ }^{4}$ Department of Pathology, UT MD Anderson Cancer Center, Houston, Texas, USA. ${ }^{5}$ Department of Biostatstistics, UT MD Anderson Cancer Center, Houston, Texas, USA. ${ }^{6}$ Department of GI Medical Oncology, UT MD Anderson Cancer Center, Houston, USA. ${ }^{7}$ Department of Interventional Radiology, UT MD Anderson Cancer Center, Houston, Texas, USA. ${ }^{8}$ Department of Epidemiology, UT MD Anderson Cancer Center, Houston, Texas, USA. ${ }^{9}$ Department of Hemopathology, UT MD Anderson Cancer Center, Houston, Texas, USA. ${ }^{10}$ Department of Radiology, Mayo Clinic, Rochester, Minnesota, USA.
Received: 19 February 2019 Accepted: 2 October 2019

Published online: 28 November 2019

\section{References}

1. Mlynarsky L, Menachem Y, Shibolet O. Treatment of hepatocellular carcinoma: Steps forward but still a long way to go. World J Hepatol. 2015; 7(3):566-74.

2. Osaki Y, Nishikawa H. Treatment of hepatocellular carcinoma in Japan over the last three decades: our experience and published work review. Hepatol Res. 2015;45:59-74.

3. Llovet JM, Villanueva A, Lachenmayer A, Finn RS. Advances in targeted therapies for hepatocellular carcinoma in the genomic era. Nature Rev. 2015;12(7):408-24.

4. Redman JM, Hill EM, AlDeghaither D, Weiner LM. Mechanisms of action of therapeutic antibodies for cancer. Mol Immunol. 2015:67(2, Part A):28-45.

5. Terme M, Ullrich E, Aymeric L, et al. IL-18 induces PD-1-dependent immunosuppression in cancer. Cancer Res. 2011;16:5393-9. https://doi.org/ 10.1158/0008-5472.CAN-11-0993 PMID: 21724589.

6. Postow M, Callahan MK, Wolchok JD. Immune checkpoint blockade in cancer therapy. J Clin Oncol. 2015;33(17):1974-82 PMID: 25605845.

7. Harding JJ, El Dika I, Abou-Alfa GK. Immunotherapy in hepatocellular carcinoma: primed to make a difference? Cancer. 2016;122:367-77.

8. Kim MJ, Choi J, Lee JS, Park J. Computed tomography findings of sorafenibtreated hepatic tumors in patients with adavanced hepatocellular carcinoma. J Gastroenterol Hepatol. 2011;26:1201-6.

9. Horger M, Lauer UM, Schraml C, et al. Early MRI response monitoring of patients with advanced hepatocellular carcinoma under treatment with the multikinase inhibitor sorafenib. BMC Cancer. 2009;9:208.

10. Park CC, Nguyen P, Hernandez C, et al. Magnetic Resonance Elastography vs Transient Elastography in detection of fibrosis and noninvasive measurement of steatosis in biopsy patients with biopsy-proven nonalcoholic fatty liver dissease. Gastroenterology. 2017;152(3):598-607.e2.

11. Venkatesh SK, Yin M, Glockner JF, et al. MR Elastography of liver tumors: preliminary results. AJR Am J Roentgenol. 2008;190:1534-40 PMID: 18492904.

12. Venkatesh SK, Yin M, Ehman RL. Magnetic resonance elastography of liver: technique, analysis and clinical applications. J Magn Reson Imaging. 2013; 37(3):544-55. https://doi.org/10.1002/jmri.23731.

13. Singh $\mathrm{S}$, et al. Diagnostic accuracy of magnetic resonance elastography in liver transplant recipients: A pooled analysis. Ann Hepatol. 2016;15(3):363-376.2.

14. Pepin KM, Chen J, Glaser KJ, Mariappan YK, Reuland B, Ziesmer S, Carter R, Ansell SM, Ehman RL, McGee KP. MR elastography derived shear stiffness--a new imaging biomarker for the assessment of early tumor response to chemotherapy. Magn Reson Med. 2014;71:1834-40 PMID: 23801372.

15. Gordic S, Ayache JB, Kennedy P, et al. Value of tumor stiffness measured with MR elastography for assessment of response of hepatocellular carcinoma to locoregional therapy. Abdom Radiol. 2017:42:1685-94 PMID: 28154910

16. Takamura T, Motosugi U, Ichikawa S, et al. Usefulness of MR elastography for detecting clinical progression of cirrhosis from child-pugh class a to B in patients with type C viral hepatitis. J Magn Reson Imaging. 2016:44:715-22 PMID: 26929192

17. Thompson SM, Wang J, Chandan VS, Glaser KJ, Roberts LR, Ehman RL, Venkatesh SK. MR Elastography of hepatocellular carcinoma: correlation of tumor stiffness with histopathology features-preliminary findings. Magn Reson Imaging. 2017;37:41-5 PMID: 27845245.

\section{Publisher's Note}

Springer Nature remains neutral with regard to jurisdictional claims in published maps and institutional affiliations. 